\title{
The association of quality of sleep with reaction time and aiming accuracy in healthy young adults
}

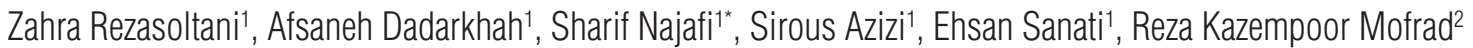

1 Aja University of Medical Sciences, Faculty of Medicine, Department of Physical Medicine and Rehabilitation

2 Shahid Beheshti University of Medical Sciences, Faculty of Medicine

\begin{abstract}
To assess the association of sleep quality with reaction time and aiming accuracy in young healthy individuals, we performed a one-group crosssectional study in an outpatient clinic of the Department of Physical Medicine and Rehabilitation at a University Hospital. Overall, 32 young men at the age range of 18 to 24 years were recruited from the university students. All participants were healthy people with no important physical or psychological problems. Quality of sleep was assessed using the Pittsburgh Sleep Quality questionnaire. Reaction time and aiming accuracy were measured with electrical reaction timer and tremometer, respectively. Quality of sleep was significantly correlated with reaction time and aiming accuracy (all p values < 0.001). In reaction time Pearson's correlation coefficients were $0.882,0.868,0.824$, and 0.894 for the association of sleep quality with simple auditory stimuli, simple visual stimuli, choice, and the number of errors, respectively. In aiming accuracy the coefficients were $0.823,0.828$, and 0.853 for total time, error time, and number of errors, respectively. To our knowledge, there has been no research published in the literature for the effect of sleep quality on aiming accuracy. Our results showed that the quality of sleep is obviously correlated with reaction time and aiming accuracy. The relations are positive and highly significant. Healthy young men with a higher quality of sleep respond faster to external stimuli and have greater concentration and aiming accuracy. Improving the quality of sleep enhances cognitive performance.
\end{abstract}

Keywords: Sleep quality, reaction time, aiming accuracy, young

\section{Introduction}

Sleep disturbance influences well-being and quality of life (O’Leary, Small, Panaite, Bylsma, \& Rottenberg, 2017) and is commonly associated with mood disorders and medical illnesses (Choudhary, Kishanrao, Dadarao Dhanvijay, \& Alam, 2016; Liu et al., 2016). Poor sleep quality increases the risk for obesity, diabetes, high blood pressure, coronary heart disease and stroke (Liu et al., 2016). Also, physical problems such as obstructive sleep apnea syndrome

Correspondence: sh.najafi@ajaums.ac.ir, (+98) 2143823476 Etemadzadeh St, Western Fatemi, Tehran, Islamic Republic of Iran, Postal code: 1411718541

Received: 3 July 2019 Accepted: 10 August 2019

Sleep and Hypnosis

Journal homepage:

http://www.sleepandhypnosis.org

ISSN:1302-1192 (Print) 2458-9101 (Online) cause sleep disorders and thereby increase mortality (Devita et al., 2018; Liu et al., 2016). Sleep disturbances are quite common in the general population suggesting the need for paying more attention (Liu et al., 2016).

The disorders can also arise in healthy population owing to a variety of reasons including occupational stress and demands (Leitaru, Kremers, Hagberg, Bjorklund, \& Kwak, 2019). Shift workers and emergency response personnel, military and transportation industry personnel frequently experience poor sleep quality (Babson, Blonigen, Boden, Drescher, \& Bonn-Miller, 2012; Giorgi, Mattei, Notarnicola, Petrucci, \& Lancia, 2018). Impaired sleep quality subsequently affects job performance, especially when a cognitive resource is needed (Giorgi et al., 2018). Consequently, the probability of accidents and job errors may increase and therefore, loss of work productivity would ensue (Liu et al., 2016). 
The relation between cognitive performance and sleep quality has been investigated in patients with physical or mental illnesses. For example, inefficient or short-time sleep, and prolonged sleep latencies impair memory and executive functions in patients with refractory focal epilepsy more than healthy people (Chakravarty et al., 2019). Longitudinal assessments in patients with obstructive sleep apnea syndrome indicated that continuous positive airway pressure improves cognitive function and psychomotor speed compared to the patients not undergoing the intervention (Devita et al., 2018). Also, long term follow-ups of patients with obstructive sleep apnea subjected to peritoneal dialysis showed that treatment with continuous positive airway pressure restores cognitive function and the quality of life (Park, Chang, \& Kang, 2018).

There has been increasing interest in the association between poor sleep quality and cognitive performance among healthy people, as well. Positive correlations have been recognized between the quality of sleep and cognitive function in elderly people (Hunter et al., 2018). Studies showed that schooling sleep hygiene improves insights, planning, attention and episodic memory in healthy old participants (de Almondes, Leonardo, \& Moreira, 2017). A study on young athletes showed that short-term sleep deprivation is negatively associated with reaction time (Taheri \& Arabameri, 2012). Overall, fewer studies have been carried out to assess the effects of sleep disturbance on cognitive performance among young healthy population.

Executive function, visio-spacial ability, language, memory, attention and arousal, decision and response time, mood and effect are among usual cognitive assessments in studies of sleep quality in healthy individuals (Della Monica, Johnsen, Atzori, Groeger, \& Dijk, 2018; Tsapanou et al., 2017). Furthermore, there are a large number of objective and subjective outcomes for measuring the quality of sleep (Benitez \& Gunstad, 2012; Farrahi Moghaddam, Nakhaee, Sheibani, Garrusi, \& Amirkafi, 2012; Forner-Cordero, Umemura, Furtado, \& Goncalves, 2018; Khosravifar, Bandi, Alavi, \& Javadi, 2015). Some variables of interest correlate with age. Mood and arousal improve, while response time slows with age (Della Monica et al., 2018). Sex also affects the results of some measurements (Della Monica et al., 2018; Morris, Rohay, \& Chasens, 2018).

Young people constitute active sub-population in any community. However, relatively few studies have examined the effects of poor sleep quality on cognitive performance of young healthy adults. The exact pattern and magnitude of the effect are uncertain and further studies have been recommended to quantify the association (Benitez \& Gunstad, 2012; Della Monica et al., 2018). In addition, evaluation of the data is complicated by a lack of uniformity in these studies. The populations, outcomes, and procedures used for the measurements vary among studies. Moreover, while aiming accuracy is critical in the evaluation of motor performance, we did not find relevant studies to assess the relation between aiming accuracy and sleep quality. It should be noticed that aiming accuracy differs between men and women, too (Sykes Tottenham, Saucier, Elias, \& Gutwin, 2005).

We conducted a study to assess the relationship between sleep quality and cognitive performance in young healthy individuals. Our hypothesis was that the quality of sleep is associated with reaction time and aiming accuracy. We also tried to minimize the confounding effect of sex and age by selecting men of a narrow age range.

\section{METHODS}

\section{Design and setting}

From June 2018 for 6 months we performed a one-group cross-sectional study. The study was conducted in an outpatient clinic of the Department of Physical Medicine and Rehabilitation at a University Hospital. The Hospital was a large referral and subspecialty center.

\section{Participants}

We recruited participants from students of medicine, nursing, and paramedics of the university. At first, students were informed through the university website. They were invited to participate in the study as volunteers. Overall, 78 students were registered as potential participants. Next, we interviewed and examined the students for their eligibility. EKG, blood, and urine chemistries were obtained to en- 
sure that the sample is drug-free. A psychiatrist interviewed individuals and two of the authors carried out detailed physical examinations and determined eligibility. Participants also filled in a questionnaire on past medical history. Nine individuals were excluded from the pool of volunteers. Of the 69 students, 32 were randomly selected and included in the study. They were instructed to attend the study examinations according to a time schedule. Three people did not attend the assessment visits and were immediately substituted with the next three volunteers. At the beginning of the visit, participants filled in the Pittsburgh Sleep Quality Index (PSQI). Then, they underwent the assessments for time reaction and aiming accuracy. All individuals gave written informed consent before the assessments.

\section{Eligibility criteria}

We included young men in the age range of 18 to 24 years. We excluded participants with a history or known diagnosis of significant or chronic diseases with impact on sleep quality such as psychiatric, neurological, endocrinological, and cardiovascular problems. Other exclusion criteria were: alcohol and nicotine use within the last three months, high caffeine intake, any history or current use of narcotics and psychiatric medications, and incomplete collection of data or the absence of needed specimens.

\section{Outcome measures}

Quality of sleep

We used the PSQI to measure sleep quality. The self-report questionnaire can be completed within 5 minutes. It serves as a standard instrument with good test-retest reliability and validity as a Cronbach's alpha of 0.83 , a sensitivity of $89.6 \%$, and specificity of $86.5 \%$. The questionnaire consists of 19 items measured on a 4 point scale ( 0 to 3 ) for assessing sleep quality within the last month (Morris et al., 2018). The items are organized into 7 subscales asking about perceived sleep quality, sleep latency, sleep duration, habitual sleep efficiency, sleep disturbances (e.g., noise), use of sleeping medication, and daytime dysfunction (sleepiness and concentration). Total score ranges between o (very good) to 21 (very poor sleep quality). A total score of more than 5 represents poor sleep quality (Gao et al., 2018; Morris et al., 2018).

In a study on the diagnostic value of PSQI, the questionnaire was translated from English to Persian and back to English (Farrahi Moghaddam et al., 2012). Then, 125 psychiatric patients and 133 controls filled in the questionnaire. The Cronbach's alpha coefficients for the total sample, group patient, and group control were $0.77,0.52$, and 0.78 , respectively. Also, PSQI scores were significantly correlated with general health questionnaire-12 $(r=0.54$, $\mathrm{p}<0.001$ ). The sensitivity and specificity of the instrument for a PSQI cutoff value of 5 in patients with insomnia were $94 \%$ and $72 \%$.

In another study, the reliability and validity of the Persian version of the PSQI and Epworth Sleepiness Scale questionnaires were evaluated in patients with depression. Cronbach's alpha of the PSQI questionnaire was 0.821 , and evaluations showed a significant correlation between the two questionnaires (Khosravifar et al., 2015).

Eventually, we decided to use PSQI because of its diagnostic accuracy, ease of use, and correlation with other instruments.

Reaction time

Visual and auditory reaction times were measured by using an electrical reaction timer (RT9110, Sinapsycho Inc.). The device provides auditory and visual color-based stimuli. The participants responded to the stimuli by pressing a button. In simple form stimulation, the participant responded to a visual or an auditory stimulus; and in choice form, the examinee was asked to respond to one type while not respond to the other. The order and timing of the stimuli were randomly scheduled by the device. At first, participants were briefed regarding the procedure. Then, they carried out some practice trial and finally performed the actual test. Each stimulus was delivered for 10 times and the average of the reaction times was calculated. We recorded the simple visual, simple auditory, and choice reaction times, as well as the number of errors.

Aiming accuracy

Assessment of aiming accuracy was carried out using a tremometer (PM-AP20881, Sinapsycho Inc.). 
The device has a metal plate with 10 different sized holes, arranged according to their diameter. Participants should insert a hand-held, metal-tipped pen into the holes from the largest to smallest one. They were asked to do their best not to contact the stylus and the wall of the holes. Each contact was alarmed by the device beeping and recorded as an error. The number of errors, duration of contacts, and the overall duration of the test was measured and saved by the tremometer. Examinees were briefed and allowed to carry out some practice trial, too.

\section{Ethical considerations}

The study was conducted in full compliance with the Helsinki Declaration. Ethics approval was obtained from the review boards of the University. All individuals gave signed consent. A study investigator explained the rationale of the research and the aims to eligible people. All participants were informed that they are free to withdraw from the study at any time. Examinees did not pay any money for the tests.

\section{Sample size and statistical analyses}

We estimated that a sample size of 26 was needed to achieve $80 \%$ statistical power to detect a standardized effect size of $50 \%$ at a two-tailed statistical significance level of 0.05 . We added more 6 volunteers to keep test powers high and included 32 participants.
Data are presented as mean (SD) for continuous and as numbers and proportions for categorical variables. The normality of the outcome variables was examined with the Kolmogorov-Smirnov test. Correlations were calculated by Pearson's coefficients and $95 \%$ confidence intervals were estimated. The level of significance was set at 0.05. Statistical analyses were done with Statistical Package for the Social Sciences, version 19.0 (IBM Inc, Chicago, IL, USA).

\section{RESULTS}

A total of 32 young men completed the study tests. Table 1 shows the baseline characteristics of the study sample. Participants were high compliant and they underwent comprehensive clinical evaluations for free. For several equivocal situations, a consensus committee decided for the diagnosis. Our analytical sample included healthy people with no obvious physical or mental disorders influencing the results of the study. We did not have missing data in our dataset. Kolmogorov-Smirnov test of normality did not show significant departure from normality for the measurements. Table 2 shows the correlations of sleep quality with reaction time and aiming accuracy. Pearson's correlation coefficients were large and highly significant. The confidence intervals for the coefficients were narrow and it may be owing to the small standard deviations in the homogenous sample.

Table 1. Baseline characteristics of the sample $(n=32)$

\begin{tabular}{|c|c|c|c|}
\hline Variable & Mean (SD) & Minimum & Maximum \\
\hline \multicolumn{4}{|l|}{ Demographic } \\
\hline Age (year) & $21.4(1.3)$ & 19 & 24 \\
\hline Weight (kg) & $70.9(8.9)$ & 56 & 90 \\
\hline Height (cm) & $176.9(5.3)$ & 167 & 194 \\
\hline Quality of sleep index & $10.5(5.6)$ & 0 & 20 \\
\hline \multicolumn{4}{|l|}{ Reaction time } \\
\hline Simple auditory (ms) & $0.23(0.07)$ & 0.12 & 0.40 \\
\hline Simple visual (ms) & $0.25(0.10)$ & 0.13 & 0.39 \\
\hline Choice (ms) & $0.30(0.08)$ & 0.14 & 0.42 \\
\hline Number of errors & $3.2(2.3)$ & 0 & 8 \\
\hline \multicolumn{4}{|l|}{ Aiming accuracy } \\
\hline Total time (ms) & $14.70(4.75)$ & 7.82 & 25.24 \\
\hline Error time (ms) & $1.78(0.91)$ & 0.41 & 3.53 \\
\hline Number of errors & $9.9(5.6)$ & 3 & 20 \\
\hline
\end{tabular}


Table 2. Correlations of quality of sleep with reaction time and aiming accuracy ( $n=32$ )

\begin{tabular}{|c|c|c|c|c|c|c|c|c|}
\hline \multirow{2}{*}{\multicolumn{2}{|c|}{ Simple auditory }} & \multicolumn{4}{|c|}{ Reaction time } & \multicolumn{3}{|c|}{ Aiming accuracy } \\
\hline & & Simple visual & Choice & $\begin{array}{l}\text { Number of } \\
\text { errors }\end{array}$ & Total time & Error time & $\begin{array}{l}\text { Number of } \\
\text { errors }\end{array}$ & \\
\hline \multirow{4}{*}{$\begin{array}{l}\text { Quality } \\
\text { of sleep } \\
\text { index }\end{array}$} & $r$ & 0.882 & 0.868 & 0.824 & 0.894 & 0.823 & 0.828 & 0.853 \\
\hline & $95 \% \mathrm{Cl}$ for $\mathrm{r}$ & 0.7700 .941 & 0.7440 .934 & 0.6670 .911 & 0.7920 .947 & 0.6650 .910 & 0.6740 .913 & 0.7180 .926 \\
\hline & $t(d f=30)$ & 10.251 & 9.574 & 7.966 & 10.928 & 7.936 & 8.088 & 8.952 \\
\hline & $p$-value & $<0.001$ & $<0.001$ & $<0.001$ & $<0.001$ & $<0.001$ & $<0.001$ & $<0.001$ \\
\hline
\end{tabular}

r: Pearson's correlation coefficient

$\mathrm{Cl}$ : Confidence Interval

df: degrees of freedom

\section{DISCUSSION}

We tried to evaluate the relationship between sleep quality and cognitive performance in a sample of young healthy men. The confounding effect of age, sex, and physical or mental disorders were eliminated by providing a homogenous sample of people. Our results showed that the quality of sleep is clearly correlated with reaction time and aiming accuracy. The relations were positive and highly significant and the correlation coefficients were larger than what we thought previously. The results seem quite plausible and in accordance with the published research regarding reaction time. However, to our knowledge, there was no study concerning the effect of sleep quality on aiming accuracy.

Possible mechanisms for the effect of poor sleep quality on reaction time is still uncertain. However, it has been suggested that sleep problems cause sleepiness, and impair memory, attention, arousal process and oculomotor function. The resulting decrease in cognitive performance impairs patient's vigilance. Moreover, sleep disturbance may affect inhibition and memory through influencing prefrontal cortex function (Choudhary et al., 2016).

Research has been done on the relation between sleep quality and reaction time among people with a variety of physical or mental disorders. In a study on 16 patients undergoing sleep restriction therapy for insomnia, there was no significant prolongation of reaction time (Whittall, Pillion, \& Gradisar, 2018). In another study on patients with obstructive sleep apnea syndrome, it was shown that prescription of positive airway pressure affects reaction time favorably by correcting the sleep disturbance (Devita et al., 2018). However, we recruited healthy people and therefore tried to concentrate on recently published research with comparable disease-free samples.

One night sleep deprivation affects reaction time. In a randomized trial, 18 college student athletes underwent measurements for power and reaction time (Taheri \& Arabameri, 2012). There was no significant difference in anaerobic peak power or mean power from the baseline, but reaction time showed significant change $(\mathrm{P}=0.003)$. The results indicated that one occasion of sleep deprivation does not impair anaerobic performance but adversely affects reaction time. Overall, our study supported the same conclusion regarding reaction time. However, we carried out an observational study with a larger sample of healthy people. Our participants did not change their steady lifestyle around the time of the study. Hence, we were able to measure the prolonged effects of sleep quality on reaction time.

In a study on 21 anesthesiologists, researchers assessed reaction time both after an overnight call and after regular working hours (Saadat et al., 2017). They measured reaction time by means of a Psychomotor Vigilance Test device comprising a handheld monitor for assessing the time to subject's response to a visual stimulus. Participants responded to numbers scrolling on the screen with 2 to $10 \mathrm{~s}$ time interval. The sample composed of 8 women and 15 men with an age range of 32-56 years. Mean reaction time decreased significantly after overnight call by $31.2 \mathrm{~ms}$ (95\% CI: 0.5, 61.9; $\mathrm{P}=0.047$ ). That study can 
be critiqued in lack of controlling confounding variables. Their inclusion criteria were wide and there were few exclusion criteria. The sample size was small and composed of heterogeneous participants of both sexes with a wide age range. Besides, there was no adjustment reported in statistical analyses, such as ANCOVA or partial correlation controlling for age and sex. While wide standards have made the study more generalizable, the confidence interval is too wide to recognize a clear main effect. Again, they assessed reaction time after one occasion of sleep deprivation and therefore they measured the shortterm effects of sleep disturbance on reaction time. While the conclusion of that study is similar to ours, there are discrepancies between the two research regarding study question, design, and sample.

In a study on 50 watchmen with 18 to 35 years of age, mental attention, and visual and auditory reaction times were evaluated one, four, and seven days after sleep restriction (Choudhary et al., 2016). Participants were allocated to two groups of normal $(n=28)$ and restricted $(n=22)$ sleep. Group restricted had less than or equal to 3 hours sleep at night. The results indicated that there was no significant difference between the two groups in the Karolinska Sleepiness Scale score and visual and auditory reaction times on the first day. However, for days 4 and 7 the score and reaction times increased significantly compared to normal sleep watchmen. It was concluded that sleep restriction has an obvious impact on attention and reaction time. Also, it was confirmed that four or more days of partial sleep restriction cumulatively impairs neurobehavioral functions and psychomotor tasks.

Overall, our results were similar to the results of that study regarding time reaction. Our participants were more alike in their ages and were more stable in their sleep quality. Thus, we measured the longterm effects of sleep quality on cognitive performance. Besides, we assessed aiming accuracy and estimated exact correlations.

To our knowledge, there is no research published in the literature for the effect of sleep quality on aiming accuracy. So, maybe this is the first article containing an exact numerical report of the correlations between quality of sleep and aiming accuracy.
Previous research has been concentrated on the influence of fatigue (Gil-Cosano, Orantes-Gonzalez, \& Heredia-Jimenez, 2019), limb preference (Sherwood, 2014), visual feedback (Sherwood \& Duffell, 2010; Yoshida, Cauraugh, \& Chow, 2004), and sex (Sykes Tottenham et al., 2005) on aiming accuracy.

\section{Strengths and limitations}

Our sample was homogenous and sufficiently large to detect important effects. The analyses were straightforward and the results were conclusive. However, we included only male participants with a narrow age range. Of course, the rationale behind the eligibility criteria was based on the published evidence. We were aimed to find if there was a large main effect of sleep quality on reaction time and aiming accuracy. Therefore, we tried to control confounding variables of sex and age when designing our study.

There is broad evidence regarding the effects of sex hormones on sleep mechanism (Morris et al., 2018). It has been suggested that the basic concept of sleep quality is different between men and women. Premenopausal women have a different circadian rhythm, sleep debt accumulates more quickly in women and the period of recovery is longer. It is likely that women show different symptoms of impaired sleep. Researchers who use PSQI have been warned regarding the effects of gender differences in the quality of sleep (Morris et al., 2018). Similarly, significant differences have been recognized between men and women in aiming accuracy (Sykes Tottenham et al., 2005).

Also, the prevalence of poor sleep quality increases with age as it has been reported frequently in the literature (Lin, Lin, \& Wang, 2019). It has been asserted that the positive relationship between age and quality of sleep is more consistent in women (Madrid-Valero, Martinez-Selva, Ribeiro do Couto, Sanchez-Romera, \& Ordonana, 2017). In a cross-sectional study of 354 participants between 20 and 99 years of age, a significant non-linear relation between reaction time and age was recognized (Blomkvist et al., 2017). The difference in mean reaction time showed that men are significantly faster than women (Blomkvist et al., 2017). 


\section{CONCLUSION}

Our results showed that the quality of sleep is obviously correlated with reaction time and aiming accuracy. The relations are positive and highly significant and the correlations are large. Healthy young men with a higher quality of sleep respond faster to external stimuli and have greater concentration and aiming accuracy. Improving the quality of sleep enhances the speed and accuracy of aiming movements and cognitive performance.

\section{Highlights}

- Quality of sleep is obviously correlated with reaction time and aiming accuracy.

\section{References}

Babson, K. A., Blonigen, D. M., Boden, M. T., Drescher, K. D., \& Bonn-Miller, M. O. (2012). Sleep quality among U.S. military veterans with PTSD: a factor analysis and structural model of symptoms. [Research Support, Non-U.S. Gov't Research Support, U.S. Gov't, Non-P.H.S.]. J Trauma Stress, 25(6), 665-674. doi: $10.1002 /$ jts.21757

Benitez, A., \& Gunstad, J. (2012). Poor sleep quality diminishes cognitive functioning independent of depression and anxiety in healthy young adults. Clin Neuropsychol, 26(2), 214-223. doi: 10.1080/13854046.2012.658439

Blomkvist, A. W., Eika, F., Rahbek, M. T., Eikhof, K. D., Hansen, M. D., Sondergaard, M., . . . Jorgensen, M. G. (2017). Reference data on reaction time and aging using the Nintendo Wii Balance Board: A cross-sectional study of 354 subjects from 20 to 99 years of age. [Research Support, Non-U.S. Gov't]. PLoS One, 12(12), e0189598. doi: 10.1371/journal.pone.0189598

Chakravarty, K., Shukla, G., Poornima, S., Agarwal, P., Gupta, A., Mohammed, A., . . . Behari, M. (2019). Effect of sleep quality on memory, executive function, and language performance in patients with refractory focal epilepsy and controlled epilepsy versus healthy controls - A prospective study. Epilepsy Behav, 92, 176-183. doi: 10.1016/j.yebeh.2018.12.028

Choudhary, A. K., Kishanrao, S. S., Dadarao Dhanvijay, A. K., \& Alam, T. (2016). Sleep restriction may lead to disruption in physiological attention and reaction time. Sleep Sci, 9(3), 207-211. doi: 10.1016/j.slsci.2016.09.001

de Almondes, K. M., Leonardo, M. E. M., \& Moreira, A. M. S. (2017). Effects of a cognitive training program and sleep hygiene for executive functions and sleep quality in healthy elderly. Dement Neuropsychol, 11(1), 69-78. doi: 10.1590/1980-57642016dn11010011

Della Monica, C., Johnsen, S., Atzori, G., Groeger, J. A., \& Dijk, D. J. (2018). Rapid Eye Movement Sleep, Sleep Continuity and Slow Wave Sleep as Predictors of Cognition, Mood, and Subjective
- The relations were positive and highly significant.

- Improving the quality of sleep enhances cognitive performance.

\section{Acknowledgments}

None.

\section{Informed consent}

Informed consent was obtained from all the participants included in the study.

\section{Conflict of Interest}

The authors declare no conflict of interest.

\section{Funding}

The authors declare that the current study was not financially supported by any institution or organization.

Sleep Quality in Healthy Men and Women, Aged 20-84 Years. Front Psychiatry, 9, 255. doi: 10.3389/fpsyt.2018.00255

Devita, M., Zangrossi, A., Marvisi, M., Merlo, P., Rusconi, M. L., \& Mondini, S. (2018). Global cognitive profile and different components of reaction times in obstructive sleep apnea syndrome: Effects of continuous positive airway pressure over time. Int J Psychophysiol, 123, 121-126. doi: 10.1016/j.ijpsycho.2017.10.003

Farrahi Moghaddam, J., Nakhaee, N., Sheibani, V., Garrusi, B., \& Amirkafi, A. (2012). Reliability and validity of the Persian version of the Pittsburgh Sleep Quality Index (PSQI-P). [Comparative Study Validation Studies]. Sleep Breath, 16(1), 79-82. doi: 10.1007/s11325-010-0478-5

Forner-Cordero, A., Umemura, G. S., Furtado, F., \& Goncalves, B. (2018). Comparison of sleep quality assessed by actigraphy and questionnaires to healthy subjects. Sleep Sci, 11(3), 141-145. doi: 10.5935/1984-0063.20180027

Gao, X., Ge, H., Jiang, Y., Lian, Y., Zhang, C., \& Liu, J. (2018). Relationship between Job Stress and 5-HT2A Receptor Polymorphisms on Self-Reported Sleep Quality in Physicians in Urumqi (Xinjiang, China): A Cross-Sectional Study. [Research Support, Non-U.S. Gov't]. Int J Environ Res Public Health, 15(5). doi: 10.3390/ijerph15051034

Gil-Cosano, J. J., Orantes-Gonzalez, E., \& Heredia-Jimenez, J. (2019). Effect of carrying different military equipment during a fatigue test on shooting performance. Eur J Sport Sci, 19(2), 186-191. doi: 10.1080/17461391.2018.1502359

Giorgi, F., Mattei, A., Notarnicola, I., Petrucci, C., \& Lancia, L. (2018). Can sleep quality and burnout affect the job performance of shift-work nurses? A hospital cross-sectional study. [Observational Study]. J Adv Nurs, 74(3), 698-708. doi: 10.1111/ jan. 13484

Hunter, J. C., Handing, E. P., Casanova, R., Kuchibhatla, M., Lutz, M. W., Saldana, S., . . . Hayden, K. M. (2018). Neighborhoods, sleep quality, and cognitive decline: Does where you live and 
how well you sleep matter? Alzheimers Dement, 14(4), 454-461. doi: 10.1016/j.jalz.2017.10.007

Khosravifar, S., Bandi, M. G., Alavi, K., \& Javadi, P. H. (2015). Evaluation of the psychometric properties of the Persian version of the Pittsburgh Sleep Quality Index in depressed patients. Electron Physician, 7(8), 1644-1652. doi: 10.19082/1644

Leitaru, N., Kremers, S., Hagberg, J., Bjorklund, C., \& Kwak, L. (2019). Associations Between Job-Strain, Physical Activity, Health Status, and Sleep Quality Among Swedish Municipality Workers. J Occup Environ Med, 61(2), e56-e6o. doi: 10.1097/ JOM.0ooooooooooo1516

Lin, K. Y., Lin, Y. C., \& Wang, H. H. (2019). Differential effects of age on quality of sleep and depression in patients receiving maintenance haemodialysis. Psychogeriatrics. doi: 10.1111/psyg.12424

Liu, Y., Wheaton, A. G., Chapman, D. P., Cunningham, T. J., Lu, H., \& Croft, J. B. (2016). Prevalence of Healthy Sleep Duration among Adults--United States, 2014. MMWR Morb Mortal Wkly Rep, 65(6), 137-141. doi: 10.15585/mmwr.mm6506a1

Madrid-Valero, J. J., Martinez-Selva, J. M., Ribeiro do Couto, B., Sanchez-Romera, J. F., \& Ordonana, J. R. (2017). Age and gender effects on the prevalence of poor sleep quality in the adult population. Gac Sanit, 31(1), 18-22. doi: 10.1016/j.gaceta.2016.05.013

Morris, J. L., Rohay, J., \& Chasens, E. R. (2018). Sex Differences in the Psychometric Properties of the Pittsburgh Sleep Quality Index. [Research Support, N.I.H., Extramural]. $J$ Womens Health (Larchmt), 27(3), 278-282. doi: 10.1089/jwh.2017.6447

O’Leary, K., Small, B. J., Panaite, V., Bylsma, L. M., \& Rottenberg, J. (2017). Sleep quality in healthy and mood-disordered persons predicts daily life emotional reactivity. [Research Support, N.I.H., Extramural]. Cogn Emot, 31(3), 435-443. doi: 10.1080/02699931.2015.1126554

Park, K. S., Chang, J. H., \& Kang, E. W. (2018). Effects of 12 months of continuous positive airway pressure therapy on cognitive function, sleep, mood, and health-related quality of life in a peritoneal dialysis patient with obstructive sleep apnea. Kidney Res Clin Pract, 37(1), 89-93. doi: 10.23876/j.krcp.2018.37.1.89

Saadat, H., Bissonnette, B., Tumin, D., Raman, V., Rice, J., Barry, N., \& Tobias, J. (2017). Effects of partial sleep deprivation on reaction time in anesthesiologists. Paediatr Anaesth, 27(4), 358362. doi: 10.1111/pan.13035

Sherwood, D. E. (2014). Aiming accuracy in preferred and non-preferred limbs: implications for programing models of motor control. Front Psychol, 5, 1236. doi: 10.3389/fpsyg.2014.01236

Sherwood, D. E., \& Duffell, B. (2010). Concurrent Visual Feedback, Practice Organization, and Spatial Aiming Accuracy in Rapid Movement Sequences. Int J Exerc Sci, 3(2), 78-91.

Sykes Tottenham, L., Saucier, D. M., Elias, L. J., \& Gutwin, C. (2005). Men are more accurate than women in aiming at targets in both near space and extrapersonal space. Percept Mot Skills, 101(1), 3-12. doi: 10.2466/pms.101.1.3-12

Taheri, M., \& Arabameri, E. (2012). The effect of sleep deprivation on choice reaction time and anaerobic power of college student athletes. Asian J Sports Med, 3(1), 15-20.

Tsapanou, A., Gu, Y., O’Shea, D. M., Yannakoulia, M., Kosmidis, M. H., Dardiotis, E., ... Scarmeas, N. (2017). Dataset on the associations between sleep quality/duration and cognitive performance in cognitively healthy older adults. Data Brief, 14, 720-723. doi: 10.1016/j.dib.2017.08.028

Whittall, H., Pillion, M., \& Gradisar, M. (2018). Daytime sleepiness, driving performance, reaction time and inhibitory control during sleep restriction therapy for Chronic Insomnia Disorder. Sleep Med, 45, 44-48. doi: 10.1016/j.sleep.2017.10.007

Yoshida, M., Cauraugh, J. H., \& Chow, J. W. (2004). Specificity of practice, visual information, and intersegmental dynamics in rapid-aiming limb movements. [Clinical Trial Randomized Controlled Trial]. $J$ Mot Behav, 36(3), 281-290. doi: 10.3200/ JMBR.36.3.281-290 